\title{
The mechanism of triptolide in the treatment of connective tissue disease-related interstitial lung disease using network pharmacology and molecular docking
}

\author{
Wen Zhu ( 1127903351@qq.com ) \\ Nanjing University of Chinese Medicine \\ Yehui Li \\ Nanjing University of Chinese Medicine \\ Junjie Zhao \\ Nanjing University of Chinese Medicine \\ Yifan Wang \\ Nanjing University of Chinese Medicine \\ Yordan Angelov Garov \\ Nanjing University of Chinese Medicine \\ Yue Wang \\ Nanjing University of Chinese Medicine
}

\section{Research Article}

Keywords: Tripterygium wilfordii, Triptolide, CTD-ILD, Network Pharmacology, Molecular docking

Posted Date: July 26th, 2021

DOI: https://doi.org/10.21203/rs.3.rs-744924/v1

License: (a) (i) This work is licensed under a Creative Commons Attribution 4.0 International License.

Read Full License 


\section{Abstract}

Background Interstitial lung disease(ILD) is associated with substantial morbidity and mortality, which is one of key systematic manifestations of connective tissue disease (CTD). Tripterygium wilfordii, known as Leigongteng in Chinese, has been applied to treat connective tissue disease-related interstitial lung disease (CTD-ILD) for many years. Methods Triptolide is key effective components from Tripterygium wilfordii. But the molecular mechanism of Triptolide for treating CTD-ILD is not yet clear. Gain insight into the molecular mechanism of Triptolide intervene CTD-ILD, we used the method of network pharmacology. And then we conducted an drug-target networks to analysis potential protein targets between Triptolide and CTD-ILD. Finally, AutoDock Vina was selected for molecular docking. Results By analysis the interaction genes of Triptolide with CTD-ILD, 242 genes were obtained. The top 10 targets of the highest enrichment scores were STAT3, AKT1, MAPK1, IL6, TP53, MAPK3, RELA, TNF, JUN, JAK2. GO and KEGG enrichment analysis exhibited that mutiple signaling pathway were involved. PI3K-Akt, multiple virus infection, cancer signaling, chemokine and apoptosis signaling pathway are the main pathways for Triptolide intervention CTD-ILD, which are related to various biological processes such as inflammation, infection, cell apoptosis and cancer. Molecular docking shows Triptolide can bind with its target protein in a good bonds by Intermolecular force. Conclusions This study preliminarily reveals the internal molecular mechanism of Triptolide can interfere CTD-ILD through multiple targets, multiple access, validated through molecular docking. Provided a research basis for the following study of pathogenesis in Triptolide treatment CTD-ILD.

\section{Introduction}

Interstitial lung disease (ILD), associated with significant morbidity and mortality, is a common manifestation in patients with connective tissue disease (CTD) [1]. CTD-ILD, which are characterized by immune-mediated tissue injury that can involve the lungs, have a mix of inflammatory and fibrosis[2]. ILD along with multicompartment lung involvement including airways, pleural and pulmonary vascular disease, which may contribute to the etiology of their respiratory impairment and potential responses to therapy[3]. Both the innate and adaptive immune system can induce the development of fibrosis[4]. Following the injury, wound-healing responses are accompanied. If sustained and deregulated, pathological fibrogenesis then occurs, whereby the rate of new collagen synthesis exceeds the rate of collagen degradation, culminating in the accumulation of collagen over time[5]. During the development and evolution of this disease, immune imbalance is the main pathological factor, and a variety of immune cells, such as T cells, B cells, macrophages, etc. are involved in the occurrence and development of this disease[6,7]. Controlling a variety of pro-inflammatory factors and restoring immune homeostasis is the main direction of inhibiting the progression of this disease from inflammation to fibrosis. Among them, FGF(Fibroblast growth factor), VEGF(Vascular endothelial growth factor), TGF(transforming growth factor), PDGF(platelet derived growth factor) were thought the critical pro-inflammatory factors[8]. Nintedanib and pirfenidone can target the above targets and play a therapeutic role in this disease. But there is currently little high-level evidence to guide the management of CTD-ILD. Novel 
approaches involving the biological agents, antifibrotic drugs, and even stem cell transplant have been introduced for CTD-ILD treatment, although specific pulmonary benefit has not been conclusive. Thus, further efforts are still urgently needed to develop novel strategies to prevent this refractory respiratory disease.

Tripterygium wilfordii is a clinically common Chinese herbal medicine, which has been used to treat clinical diseases for more than 2000 years in China. Because of its good immunosuppressive effect in the fields of metabolic diseases[9], kidney diseases[10], rheumatic diseases[11], inflammatory bowel diseases[12] and so on[13], Tripterygium wilfordii hance has been paid much attention by scholars at home and abroad. Triptolide is a major active natural product isolated from the medicinal plant Tripterygium wilfordii, has exhibited to have good anti-inflammatory, immunosuppressive and antifibrosis therapeutic effects[9], but its molecular mechanism of action is still unknown. Network pharmacology is an emerging discipline based on the network of disease-gene-drug targets[14], based on network interaction to study the basic biological knowledge of TCM can provide a deep insight or scientific evidence for the discovery of TCM, and help us to clarify the pharmacological mechanism of active ingredients of TCM at the level of biomolecule[15]. Network pharmacology is gradually becoming a holistic and efficient tool to describe the complex interactions between drugs and biological systems including the human organs, diseases, metabolic pathways, and target proteins from a network perspective [16].

In this study, we constructed a network pharmacological model of Triptolide and systematically analyzed the potential anti-CTD-ILD mechanism of Triptolide. Our studies revealed the potential mechanisms which may contribute to the occur of CTD-ILD. To confirm the predicted result, we provide a molecular docking model with potential targets and known targets. The detailed procedures can be seen in Figure. 1.

\section{Materials And Methods}

\section{Screening of triptoline-related disease targets}

All targets of Triptolide were gathered by using the following five database: Traditional Chinese Medicine Systems Pharmacology Database and Analysis Platform (http://lsp.nwu.edu.cn/index.php)[17], the Comparative Toxicogenomics Database(CTD)[18] (http://ctdbase. org/), GeneCards database (https://www.genecards.org)[19,20], STITCH (http://stitch.embl.de/)[21] and SymMap database (https://www.symmap.org/)[22]. Organism equal to Homo sapiens was limited. After remove the duplicated genes, related genes were returned.

\section{Gene screening of CTD-ILD related targets}

Using "Connective Tissue Disease associated with Interstitial Lung Diseases" as the search keywords, we searched OMIM database (http://www.omim.org), Genebank database (https://www.ncbi.nlm.nih.gov/genbank), GeneCards database (https://www.genecards.org) to excavate potential targets associated with CTD-ILD. Access the DrugBank database (https://www.drugbank.ca) to 
find drug targets for approved intervention CTD-ILD [23]. The 4 disease database targets were combined and the repeat value was deleted to get the CTD-ILD related targets.

\section{Construction of protein-protein interaction (PPI) network}

Using R X 64 4.0.2 software, the intersection of the Triptolide target and related CTD-ILD target was obtained冈then the venn diagram was drawn. Intersection targets were extracted and submitted to STRING database (https://string-db.org) to build a protein-protein interaction(PPI) network[24]. The species type was set to "Homo sapiens", the minimum interaction threshold was set to "highest confidence" ( $>0.9)$, and the rest were set as the default. Finally the PPI result were imported into Cytoscape 3.8.0 software to construct a PPI network.

\section{Hub gene analysis}

Hub gene of PPI network of Triptolide against CTD-ILD were calculated by MCC algorithm in Cytohubba plugin of CytoScape[25], then the related protein targets network was constructed. Finally, the core targets of top 10 were also exhibited.

\section{GO and KEGG enrichment analysis}

In order to clarify the role of target proteins interacting with Triptolide target genes in gene function and signaling pathways, we conducted GO and KEGG bioenrichment analysis of potential targets of Triptolide intervention CTD-ILD by R Software (R 4.0.2 for Windows). Save the data results and use R software for visual analysis.

\section{Molecular docking}

Molecular docking was performed among the top 5 potential target proteins and known 4 relative proteins of ILD with Triptolide. The 3D structure of the target protein was downloaded from the PDB database (https://www.rcsb.org). The water molecules and the original ligands were removed from target protein through PyMOL, Later, the target proteins were imported into AutoDock Tools 1.5.6 for hydrogenation, charge calculation, and non-polar hydrogen combination, and then the result were stored in PDBQT format. Set the size of Grid Box to $40 \times 40 \times 40$. Finally, run AutoDock Vina using CMD command characters for molecular docking [26], and use PyMOL to visualize the results.

\section{Results}

\section{Acquisition of the main protein targets of Triptolide}

By searching the following databases: TCMSP, CTD, GeneCards, STITCH and SymMap database,confining the result to "Homo sapiens" $₫ 702$ genes related with Triptolide were collected by using the term "Connective Tissue Disease associated Interstitial Lung Diseases".

\section{Acquisition of CTD-ILD related targets}


In Genecards database, the higher the Score value is, the closer the relationship is to the disease. When there are too many targets, the target with a Score greater than the median is a CTD-ILD related target. The maximum Score of the target obtained by GeneCards is 166.33 , the minimum Score is 0.38 , and the median is 17.64. Therefore, the genetic target with a Score of $>17.64$ is considered as CTD-ILD related target.Then 1764 targets are obtained after screening. After searching the GenBank database, we found 86 related genes. A total of 1294 targets were obtained from the Genemap database. The DrugBank database search is complemented \and about 12 targets for Nintedanib were obtained. Results were merged and the duplicate genes were eliminated, then 2860 CTD-ILD related targets were obtained.

\section{Venn diagram and PPI network construction}

The intersection of Triptolide targets and CTD-ILD disease targets was taken, and venn diagram was drawn by R software to obtain 271 intersection targets (Figure. 2). The target proteins that act with its corresponding ingredients were submitted to STRING version 11.0 (http://string-db.org/) for PPI network construction, and high confidence of protein interaction data with a score $>0.9$ was selected . Removing free proteins that do not interact,There were 242 shared proteins between Triptolide and CTD-ILD. The protein-protein interactions networks suggested that 242 proteins and 1628 interactions (edges) could potentially interact in the intersection targets between Triptolide and CTD-ILD (Figure. 3A).

\section{Screening of Hub gene and Topological network analysis}

Based on Cytohubba, the plug-in of Cytoscape, the Hub gene was screened in the interaction network. We used MCC algorithm to find out the top 10 Hub genes of target proteins, and the Hub gene network diagram was constructed. Based on network topological analysis, the average betweenness centrality was 0.01 and the average degree of freedom was 13.45 , signaling a hub connectivity between genes. $A$ total of 98 genes produced betweenness centrality and degree of freedom values above the meanखbut only the top 10 genes are exhibited(Table 1).We can come to a conclusion that STAT3, AKT1, MAPK1, IL6, TP53, MAPK3, RELA, TNF, JUN, JAK2 is of the important targets for Triptolide intervention CTDILD(Figure. 3B).

Table 1 | Target proteins with potentially critical roles in Triptolide treatment of CTD-ILD. 


\begin{tabular}{llll} 
NO. & Gene abbreviation & Betweenness centrality & Degree \\
\hline 1 & STAT3 & 0.093621689 & 63 \\
\hline 2 & MAPK1 & 0.068443793 & 54 \\
\hline 3 & AKT1 & 0.065861966 & 54 \\
\hline 4 & IL6 & 0.080875215 & 52 \\
\hline 5 & TP53 & 0.078781834 & 51 \\
\hline 6 & MAPK3 & 0.034771135 & 50 \\
\hline 7 & RELA & 0.042217185 & 46 \\
\hline 8 & JUN & 0.035870028 & 42 \\
\hline 9 & TNF & 0.031940891 & 42 \\
\hline 10 & JAK2 & 0.028176151 & 38
\end{tabular}

\section{GO and KEGG analysis}

The GO and KEGG enrichment analysis of Triptolide intervention CTD-ILD related targets was carried out with $\mathrm{R}$ software, and the results were visualized. Topological network schematic of target proteins in enriched molecular functions analyzed by enrichMap in the clusterProfiler package are listed(Figure. 4). The GO enrichment analysis results obtained a total of 3261 items, The top 20 significantly enriched terms in $\mathrm{BP}, \mathrm{MF}$, and $\mathrm{CC}$ categories were selected, according to $\mathrm{P}<0.05$, P-values were corrected using the Benjamini-Hochberg procedure. In biological processes category the target proteins were mainly involved in Apoptosis, cell oxidative stress, inflammatory response, etc. In the MF category, the target proteins were

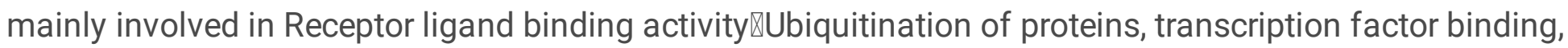
Cytokine activity. In the CC category, the target proteins were classified into plasma membrane and cell surface冈endoplasmic reticulum lumen, etc.

There were $174 \mathrm{KEGG}$ enrichment items in total, and the top 20 items were screened according to the KEGG analysis with BH-corrected P-values $<0.05 \rrbracket$ mainly included PI3K-Akt signaling pathway,multiple virus infection, chemokine signaling pathway and apoptosis signaling pathway et al. The first 20 related enrichment results were visualized based on GO and KEGG enrichment results (Figure. 5).

\section{Molecular docking}

Molecular docking was conducted between Triptolide冈the core components of Tripterygium wilfordii and the key targets. Then the docking results showed that Triptolide could be bound into the docking pocket, with good docking activity between the target proteins. As we tested the following potential target proteins(Fig. 6): TP53(PDB: 3DAB), MAPK1(PDB: 3W55), STAT3 (PDB: 6NUQ), AKT1(PDB: 6NPZ) and IL6(PDB: 2IL6), which were high-degree nodes in the interaction network, suggesting they play a critical role in the response to Triptolide in CTD-ILD. Triptolide bound to TP53 by forming hydrogen bonds with 


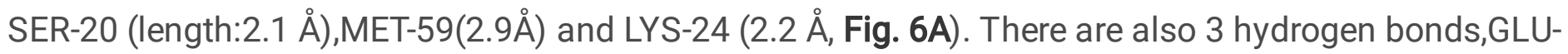

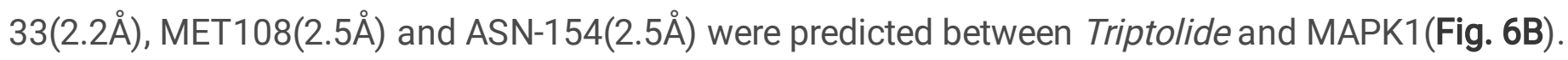
Similarly, Triptolide was predicted to dock into the binding pocket of STAT3 via hydrogen bonds SER-

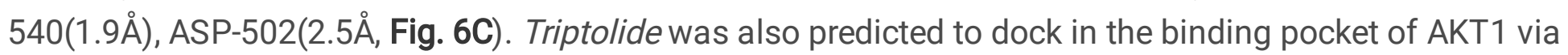

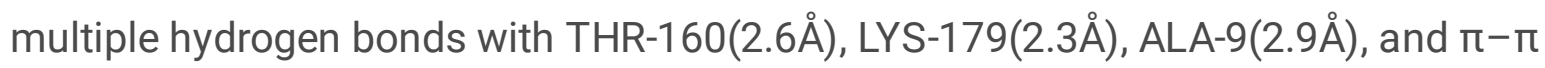
interactions with PHE-161(3.7 A, Fig. 6D). Lastly, Triptolide was predicted to dock into the pocket of IL-6 via a single hydrogen bonds with THR-164 at a distance of $2.4 \AA$ (Fig. 6E). Futhermore, known protein targets of ILD(FGF, PDGF, VEGF, TGF) was also conducted molecule docking with Triptolide, of whom binding energies were all less than $-5 \mathrm{kcal} \cdot \mathrm{mol}^{-1}$. It is suggested that Triptolide may affect its function by competitively inhibiting the binding of the docking pocket to the target receptor and play an important role in the treatment of CTD-ILD.

\section{Discussion}

Network pharmacology, as a cutting-edge approach provides a full or partial understanding of the principles of network theory and systems biology[27]. This approach has been used to study the pathway "compound-proteins/genes-disease" in a way that captures the complexities among biological systems, drugs, and diseases from a network perspective. Therefore, the network pharmacology research method is used to predict the interrelationship network between drugs and diseases in the study of different fields such as discover new drugs[28] elaborate pharmacological mechanism[29], and explore new targets[30]. In our study, topological analysis of drug-disease network was conducted, and corresponding molecular docking studies were conducted to enhance the reliability of target prediction conclusions.

In the present report, we predict interactions between Triptolide and its potential protein targets by integrating information from publicly available databases about CTD-ILD, as well as to elucidate the numerous signaling pathways and networks in which Triptolide targets participate. We also performed docking studies to predict specific interactions between Triptolide and its predicted protein targets. The molecular docking results of Triptolide showed that hydrogen bonding and $\pi-\pi$ stacking were the main forms of interaction. Pathway analysis suggested that Triptolide regulates the activation of mainly included PI3K-Akt signaling pathway, multiple virus infection, chemokine signaling pathway, apoptosis signaling pathway, TNF signaling pathway and IL-17 signaling pathway in CTD-ILD(Fig. 7).

The PI3K-Akt signaling pathway, TNF signaling pathway, and IL-17 signaling pathway are known as inflammation-related signaling pathways. In addition, Activating the PI3K-Akt and the mammalian target of rapamycin (mTOR) signaling pathway can inhibits autophagy of bronchial epithelial cells and exacerbates lung damage and fibrosis[31]. Accordingly, inhibiting this pathway can attenuates mitochondrial-dependent apoptosis, endoplasmic reticulum stress, and inflammation in acute lung injury[32]. TNF signaling pathway plays an important role in a variety of connective tissue diseases. Tumor necrosis factor (TNF) receptors and their corresponding cytokine ligands are involved in many aspects of immune functional [33], activation of TNF signaling pathway has been found to induce interstitial lung disease[34]. Interleukin(IL)-17 signal pathway demonstrated pro-inflammatory effects in 
chronic inflammation and autoimmune diseases, and showed pathogenicity in pulmonary fibrosis, are potent therapeutic targets for the treatment of fibroproliferative lung diseases[35]. Interestingly, Signaling pathways are not isolated from each other, such as the IL-17 and Akt pathways have a promoting effect. One study confirmed that IL-17A can inhibit autophagy in keratinocytes by activating the PI3K/Akt/mTOR signalling pathway[36]. Multiple studies have found that viral infection can induce the formation of hyaluronic acid[37], release a variety of inflammatory cytokines[38], associated with acute lung interstitial injury[39], is a costimulatory factor in the progression of pulmonary interstitial disease[40]. Apoptosis was thought to be the only regulated cell death mechanism[41], Regulation of cell death is the main mechanism for eliminating damaged, infected or excess cells, which is also found to be related to the occurrence of ILD[42].

Experimental studies have found that Triptolide has shown good anti-inflammatory and immunosuppressive effects in the treatment of many diseases. Studies have verified that Triptolide can attenuates inflammatory response, lung injury and kidney damage in multiple autoimmune diseases by inhibiting NF-KB, vascular cell adhesion molecule-1, IL-1, IL-6, IL-17 and TNF-a,through interfere NFKB[43,44], PI3K-AKT-mTOR and apoptosis singnal pathway et al[45]. So we think that Triptolide may play a role in the treatment of CTD-ILD through the above pathway.

Although the therapeutic effect of tripterysium glycosides in the treatment of CTD-ILD has been satisfactory, the mechanism of action of tripterysium glycosides in the treatment of CTD-ILD has been rarely studied. This paper based on the network pharmacology, and molecular docking technology discussed the the molecular mechanism of Triptolide, an element of tripterysium glycosides, intervene CTD-ILD. The results prove that Triptolide can interference the process of CTD-ILD,through multiple targets, multiple access, the top 10 targets are STAT3, AKT1, MAPK1, IL6, TP53, MAPK3, RELA, TNF, JUN, JAK2.

In view of the lack of relevant experimental studies on the treatment of CTD-ILD by Tripterygium wilfordii. Although network pharmacology is a quick and efficient method for predicting multiple drug targets in complex diseases, it is still necessary to verify the predicted targets using in vitro experiments.

\section{Conclusion}

The present study analyzed the mechanisms underlying the therapeutic effect of Triptolide in CTD-ILD using network pharmacology. Our findings revealed that Triptolide exerts pharmacological effects in CTDILD in a multicomponent-multitarget-multipathway manner, including Apoptosis, cell oxidative stress, inflammatory response and so on. Our findings offer a reference for further investigation of the mechanism underlying the therapeutic effect of Triptolide in CTD-ILD.

\section{Declarations}

\section{Data Availability Statement}


The original contributions presented in the study are included in the article/Supplementary Material, further inquiries can be directed to the corresponding authors upon reasonable request..

\section{Acknowledgement}

Not applicable.

\section{Author Contributions}

WZ and YL designed this study and performed the online database search. YW and JZ all contributed to the data collection and data analysis. YL and WZ prepared the original draft. YW finished the revision of the manuscript. YG polished up the whole article. All authors have read and approved the final manuscript.

\section{Funding}

This work was supported by the National Natural Science Foundation of China (grant 81774274, grant 81973769).

\section{Conflict of Interest}

The authors declare that the research was conducted in the absence of any commercial or financial relationships that could be construed as a potential conflict of interest.

\section{Ethics approval and consent to participate}

Not applicable.

\section{Consent to publication}

All authors have read and agreed to the published version of the manuscript.

\section{Competing interests}

The authors declared that this work was conducted in the absence of any commercial or fnancial relationship that could be construed as a potential confict of interest.

\section{References}

1. Gao, Y.; Moua, T. Treatment of the Connective Tissue Disease-Related Interstitial Lung Diseases: A Narrative Review. Mayo Clin. Proc. 2020, 95, 554-573, doi:10.1016/j.mayocp.2019.07.007.

2. $\quad$ Fischer, A.; Antoniou, K.M.; Brown, K.K.; Cadranel, J.; Corte, T.J.; du Bois, R.M.; Lee, J.S.; Leslie, K.O.; Lynch, D.A.; Matteson, E.L.; et al. An Official European Respiratory Society/American Thoracic Society 
Research Statement: Interstitial Pneumonia with Autoimmune Features. Eur. Respir. J. 2015, 46, 976-987, doi:10.1183/13993003.00150-2015.

3. Gutsche, M.; Rosen, G.D.; Swigris, J.J. Connective Tissue Disease-Associated Interstitial Lung Disease: A Review. Curr Respir Care Rep 2012, 1, 224-232, doi:10.1007/s13665-012-0028-7.

4. Spagnolo, P.; Distler, O.; Ryerson, C.J.; Tzouvelekis, A.; Lee, J.S.; Bonella, F.; Bouros, D.; HoffmannVold, A.-M.; Crestani, B.; Matteson, E.L. Mechanisms of Progressive Fibrosis in Connective Tissue Disease (CTD)-Associated Interstitial Lung Diseases (ILDs). Ann. Rheum. Dis. 2020, annrheumdis-2020-217230, doi:10.1136/annrheumdis-2020-217230.

5. Wynn, T.A. Cellular and Molecular Mechanisms of Fibrosis. J Pathol 2008, 214, 199-210, doi:10.1002/path.2277.

6. Kolahian, S.; Fernandez, I.E.; Eickelberg, O.; Hartl, D. Immune Mechanisms in Pulmonary Fibrosis. Am J Respir Cell Mol Bio/ 2016, 55, 309-22, doi:10.1165/rcmb.2016-0121TR.

7. Todd, N.W.; Scheraga, R.G.; Galvin, J.R.; lacono, A.T.; Britt, E.J.; Luzina, I.G.; Burke, A.P.; Atamas, S.P. Lymphocyte Aggregates Persist and Accumulate in the Lungs of Patients with Idiopathic Pulmonary Fibrosis. J Inflamm Res 2013, 6, 63-70, doi:10.2147/jir.s40673.

8. Hoffmann-Vold, A.-M.; Weigt, S.S.; Saggar, R.; Palchevskiy, V.; Volkmann, E.R.; Liang, L.L.; Ross, D.; Ardehali, A.; Lynch, J.P.; Belperio, J.A. Endotype-Phenotyping May Predict a Treatment Response in Progressive Fibrosing Interstitial Lung Disease. EBioMedicine 2019, 50, 379-386, doi:10.1016/j.ebiom.2019.10.050.

9. Chen, S.R.; Dai, Y.; Zhao, J.; Lin, L.; Wang, Y.; Wang, Y. A Mechanistic Overview of Triptolide and Celastrol, Natural Products from Tripterygium Wilfordii Hook F. Front Pharmaco/ 2018, 9, 104, doi:10.3389/fphar.2018.00104.

10. Zhou, Y.; Hong, Y.; Huang, H. Triptolide Attenuates Inflammatory Response in Membranous Glomerulo-Nephritis Rat via Downregulation of NF-KB Signaling Pathway. Kidney Blood Press Res 2016, 41, 901-910, doi:10.1159/000452591.

11. Fan, D.; He, X.; Bian, Y.; Guo, Q.; Zheng, K.; Zhao, Y.; Lu, C.; Liu, B.; Xu, X.; Zhang, G.; et al. Triptolide Modulates TREM-1 Signal Pathway to Inhibit the Inflammatory Response in Rheumatoid Arthritis. Int $J$ Mol Sci 2016, 17, 498, doi:10.3390/ijms17040498.

12. Li, Y.; Yu, C.; Zhu, W.M.; Xie, Y.; Qi, X.; Li, N.; Li, J.S. Triptolide Ameliorates IL-10-Deficient Mice Colitis by Mechanisms Involving Suppression of IL-6/STAT3 Signaling Pathway and down-Regulation of IL-17. Mol Immunol 2010, 47, 2467-74, doi:10.1016/j.molimm.2010.06.007.

13. Yuan, K.; Li, X.; Lu, Q.; Zhu, Q.; Jiang, H.; Wang, T.; Huang, G.; Xu, A. Application and Mechanisms of Triptolide in the Treatment of Inflammatory Diseases-A Review. Front Pharmacol 2019, 10, 1469, 
doi:10.3389/fphar.2019.01469.

14. Liu, C.; Liu, R.; Fan, H.; Xiao, X.; Chen, X.; Xu, H.; Lin, Y. Network Pharmacology Bridges Traditional Application and Modern Development of Traditional Chinese Medicine. Chin. Herb. Med. 2015, 7, 3-17.

15. Yang, M.; Chen, J.; Xu, L.; Shi, X.; Zhou, X.; An, R.; Wang, X. A Network Pharmacology Approach to Uncover the Molecular Mechanisms of Herbal Formula Ban-Xia-Xie-Xin-Tang. Evid Based Complement Altern. Med 2018, 2018, 4050714, doi:10.1155/2018/4050714.

16. Zhang, Y.; Mao, X.; Guo, Q.; Lin, N.; Li, S. Network Pharmacology-Based Approaches Capture Essence of Chinese Herbal Medicines. Chin. Herb. Med. 2016, 8, 107-116.

17. Ru, J.; Li, P.; Wang, J.; Zhou, W.; Li, B.; Huang, C.; Li, P.; Guo, Z.; Tao, W.; Yang, Y.; et al. TCMSP: A Database of Systems Pharmacology for Drug Discovery from Herbal Medicines. J Cheminform 2014, 6, 13, doi:10.1186/1758-2946-6-13.

18. Davis, A.P.; Murphy, C.G.; Saraceni-Richards, C.A.; Rosenstein, M.C.; Wiegers, T.C.; Mattingly, C.J. Comparative Toxicogenomics Database: A Knowledgebase and Discovery Tool for Chemical-GeneDisease Networks. Nucleic Acids Res 2009, 37, D786-92, doi:10.1093/nar/gkn580.

19. Safran, M.; Solomon, I.; Shmueli, O.; Lapidot, M.; Shen-Orr, S.; Adato, A.; Ben-Dor, U.; Esterman, N.; Rosen, N.; Peter, l.; et al. GeneCards 2002: Towards a Complete, Object-Oriented, Human Gene Compendium. Bioinformatics 2002, 18, 1542-3, doi:10.1093/bioinformatics/18.11.1542.

20. Safran, M.; Dalah, I.; Alexander, J.; Rosen, N.; Iny Stein, T.; Shmoish, M.; Nativ, N.; Bahir, I.; Doniger, T.; Krug, H.; et al. GeneCards Version 3: The Human Gene Integrator. Database Oxf. 2010, 2010, baq020, doi:10.1093/database/baq020.

21. Kuhn, M.; Szklarczyk, D.; Franceschini, A.; Campillos, M.; von Mering, C.; Jensen, L.J.; Beyer, A.; Bork, P. STITCH 2: An Interaction Network Database for Small Molecules and Proteins. Nucleic Acids Res 2010, 38, D552-6, doi:10.1093/nar/gkp937.

22. Wu, Y.; Zhang, F.; Yang, K.; Fang, S.; Bu, D.; Li, H.; Sun, L.; Hu, H.; Gao, K.; Wang, W.; et al. SymMap: An Integrative Database of Traditional Chinese Medicine Enhanced by Symptom Mapping. Nucleic Acids Res 2019, 47, D1110-d1117, doi:10.1093/nar/gky1021.

23. Wishart, D.S.; Feunang, Y.D.; Guo, A.C.; Lo, E.J.; Marcu, A.; Grant, J.R.; Sajed, T.; Johnson, D.; Li, C.; Sayeeda, Z.; et al. DrugBank 5.0: A Major Update to the DrugBank Database for 2018. Nucleic Acids Res 2018, 46, D1074-d1082, doi:10.1093/nar/gkx1037.

24. Szklarczyk, D.; Gable, A.L.; Lyon, D.; Junge, A.; Wyder, S.; Huerta-Cepas, J.; Simonovic, M.; Doncheva, N.T.; Morris, J.H.; Bork, P.; et al. STRING V11: Protein-Protein Association Networks with Increased Coverage, Supporting Functional Discovery in Genome-Wide Experimental Datasets. Nucleic Acids Res 2019, 47, D607-d613, doi:10.1093/nar/gky1131.

Page $11 / 19$ 
25. Chin, C.H.; Chen, S.H.; Wu, H.H.; Ho, C.W.; Ko, M.T.; Lin, C.Y. CytoHubba: Identifying Hub Objects and Sub-Networks from Complex Interactome. BMC Syst Biol 2014, 8 Supp/ 4, S11, doi:10.1186/1752-0509-8s4-s11.

26. Trott, O.; Olson, A.J. AutoDock Vina: Improving the Speed and Accuracy of Docking with a New Scoring Function, Efficient Optimization, and Multithreading. J Comput Chem 2010, 31, 455-61, doi:10.1002/jcc.21334.

27. Li, X.J.; Jiang, Z.Z.; Zhang, L.Y. Triptolide: Progress on Research in Pharmacodynamics and Toxicology. J Ethnopharmaco/ 2014, 155, 67-79, doi:10.1016/j.jep.2014.06.006.

28. Zhou, W.; Wang, Y.; Lu, A.; Zhang, G. Systems Pharmacology in Small Molecular Drug Discovery. Int J Mol Sci 2016, 17, 246, doi:10.3390/ijms17020246.

29. Ding, M.; Ma, W.; Wang, X.; Chen, S.; Zou, S.; Wei, J.; Yang, Y.; Li, J.; Yang, X.; Wang, H.; et al. A Network Pharmacology Integrated Pharmacokinetics Strategy for Uncovering Pharmacological Mechanism of Compounds Absorbed into the Blood of Dan-Lou Tablet on Coronary Heart Disease. $J$ Ethnopharmaco/ 2019, 242, 112055, doi:10.1016/j.jep.2019.112055.

30. Guo, Y.; Nie, Q.; MacLean, A.L.; Li, Y.; Lei, J.; Li, S. Multiscale Modeling of Inflammation-Induced Tumorigenesis Reveals Competing Oncogenic and Oncoprotective Roles for Inflammation. Cancer Res 2017, 77, 6429-6441, doi:10.1158/0008-5472.can-17-1662.

31. Cong, L.H.; Li, T.; Wang, H.; Wu, Y.N.; Wang, S.P.; Zhao, Y.Y.; Zhang, G.Q.; Duan, J. IL-17A-Producing T Cells Exacerbate Fine Particulate Matter-Induced Lung Inflammation and Fibrosis by Inhibiting PI3K/Akt/MTOR-Mediated Autophagy. J Cell Mol Med 2020, 24, 8532-8544, doi:10.1111/jcmm.15475.

32. Luo, X.; Lin, B.; Gao, Y.; Lei, X.; Wang, X.; Li, Y.; Li, T. Genipin Attenuates Mitochondrial-Dependent Apoptosis, Endoplasmic Reticulum Stress, and Inflammation via the PI3K/AKT Pathway in Acute Lung Injury. Int Immunopharmaco/ 2019, 76, 105842, doi:10.1016/j.intimp.2019.105842.

33. Meylan, F.; Siegel, R.M. TNF Superfamily Cytokines in the Promotion of Th9 Differentiation and Immunopathology. Semin. Immunopathol. 2017, 39, 21-28, doi:10.1007/s00281-016-0612-y.

34. Wu, E.K.; Henkes, Z.I.; McGowan, B.; Bell, R.D.; Velez, M.J.; Livingstone, A.M.; Ritchlin, C.T.; Schwarz, E.M.; Rahimi, H. TNF-Induced Interstitial Lung Disease in a Murine Arthritis Model: Accumulation of Activated Monocytes, Conventional Dendritic Cells, and CD21(+)/CD23(-) B Cell Follicles Is Prevented with Anti-TNF Therapy. J. Immunol. Baltim. Md 1950 2019, 203, 2837-2849, doi:10.4049/jimmunol.1900473.

35. Mi, S.; Li, Z.; Yang, H.Z.; Liu, H.; Wang, J.P.; Ma, Y.G.; Wang, X.X.; Liu, H.Z.; Sun, W.; Hu, Z.W. Blocking IL-17A Promotes the Resolution of Pulmonary Inflammation and Fibrosis via TGF-Beta1-Dependent and Independent Mechanisms. J Immuno/ 2011, 187, 3003-14, doi:10.4049/jimmunol.1004081. 
36. Varshney, P.; Saini, N. PI3K/AKT/MTOR Activation and Autophagy Inhibition Plays a Key Role in Increased Cholesterol during IL-17A Mediated Inflammatory Response in Psoriasis. Biochim Biophys Acta Mol Basis Dis 2018, 1864, 1795-1803, doi:10.1016/j.bbadis.2018.02.003.

37. Reeves, S.R.; Barrow, K.A.; Rich, L.M.; White, M.P.; Shubin, N.J.; Chan, C.K.; Kang, I.; Ziegler, S.F.; Piliponsky, A.M.; Wight, T.N.; et al. Respiratory Syncytial Virus Infection of Human Lung Fibroblasts Induces a Hyaluronan-Enriched Extracellular Matrix That Binds Mast Cells and Enhances Expression of Mast Cell Proteases. Front Immuno/ 2019, 10,3159, doi:10.3389/fimmu.2019.03159.

38. Seki, E.; Yoshizumi, M.; Tanaka, R.; Ryo, A.; Ishioka, T.; Tsukagoshi, H.; Kozawa, K.; Okayama, Y.; Okabe-Kado, J.; Goya, T.; et al. Cytokine Profiles, Signalling Pathways and Effects of Fluticasone Propionate in Respiratory Syncytial Virus-Infected Human Foetal Lung Fibroblasts. Cell Biol Int 2013, 37, 326-39, doi:10.1002/cbin.10044.

39. Wang, K.; Lai, C.; Li, T.; Wang, C.; Wang, W.; Ni, B.; Bai, C.; Zhang, S.; Han, L.; Gu, H.; et al. Basic Fibroblast Growth Factor Protects against Influenza A Virus-Induced Acute Lung Injury by Recruiting Neutrophils. J Mol Cell Bio/ 2018, 10, 573-585, doi:10.1093/jmcb/mjx047.

40. Vannella, K.M.; Moore, B.B. Viruses as Co-Factors for the Initiation or Exacerbation of Lung Fibrosis. Fibrogenesis Tissue Repair 2008, 1, 2, doi:10.1186/1755-1536-1-2.

41. Sauler, M.; Bazan, I.S.; Lee, P.J. Cell Death in the Lung: The Apoptosis-Necroptosis Axis. Annu. Rev. Physiol. 2019, 81, 375-402, doi:10.1146/annurev-physiol-020518-114320.

42. Mahavadi, P.; Korfei, M.; Henneke, I.; Liebisch, G.; Schmitz, G.; Gochuico, B.R.; Markart, P.; Bellusci, S.; Seeger, W.; Ruppert, C.; et al. Epithelial Stress and Apoptosis Underlie Hermansky-Pudlak SyndromeAssociated Interstitial Pneumonia. Am. J. Respir. Crit. Care Med. 2010, 182, 207-219, doi:10.1164/rccm.200909-14140C.

43. Huang, J.; Zhou, L.; Wu, H.; Pavlos, N.; Chim, S.M.; Liu, Q.; Zhao, J.; Xue, W.; Tan, R.X.; Ye, J.; et al. Triptolide Inhibits Osteoclast Formation, Bone Resorption, RANKL-Mediated NF-ҚB Activation and Titanium Particle-Induced Osteolysis in a Mouse Model. Mol Cell Endocrinol 2015, 399, 346-53, doi:10.1016/j.mce.2014.10.016.

44. Zhou, Y.; Hong, Y.; Huang, H. Triptolide Attenuates Inflammatory Response in Membranous Glomerulo-Nephritis Rat via Downregulation of NF-KB Signaling Pathway. Kidney Blood Press Res 2016, 41, 901-910, doi:10.1159/000452591.

45. Dai, J.; Sun, Y.; Chen, D.; Zhang, Y.; Yan, L.; Li, X.; Wang, J. Negative Regulation of PI3K/AKT/MTOR Axis Regulates Fibroblast Proliferation, Apoptosis and Autophagy Play a Vital Role in Triptolide-Induced Epidural Fibrosis Reduction. Eur J Pharmaco/ 2019, 864, 172724, doi:10.1016/j.ejphar.2019.172724.

\section{Figures}




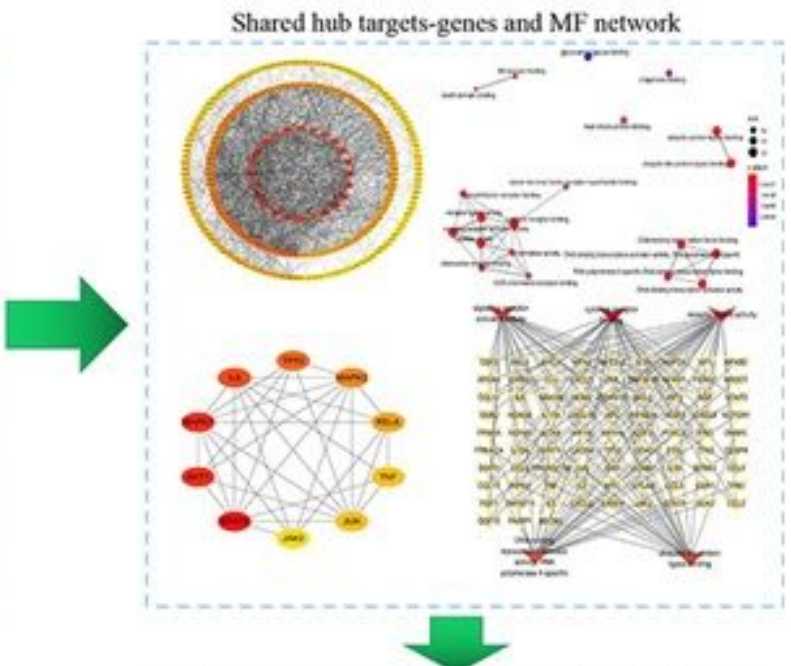

Molecular docking results

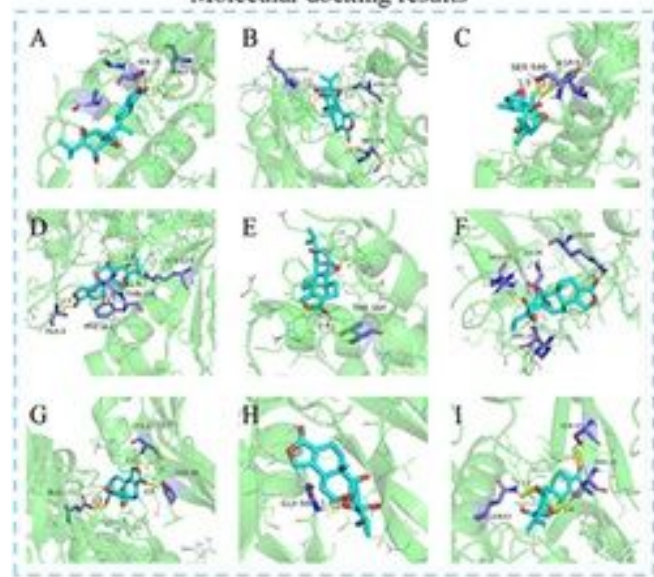

Go and kegg analysis of shared protein, targets

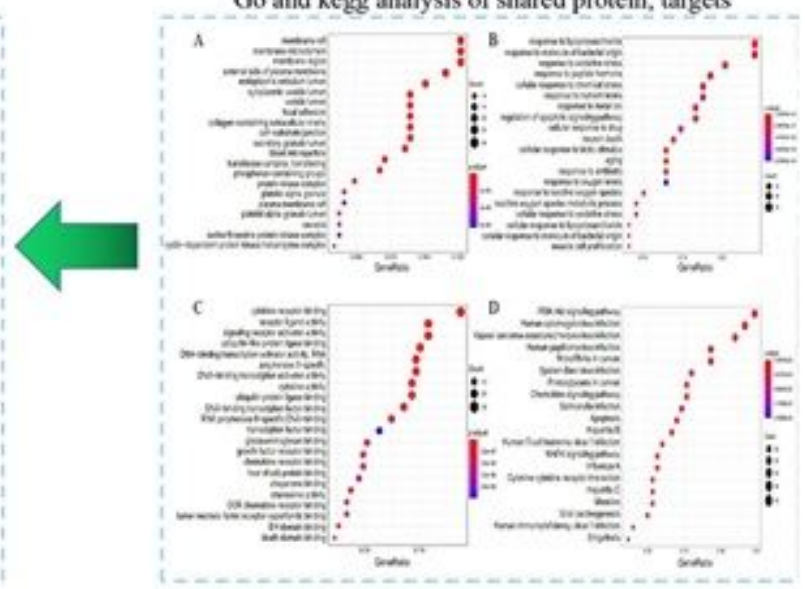

Figure 1

Workflow of the network pharmacology to identify Triptolide targets in CTD-ILD.

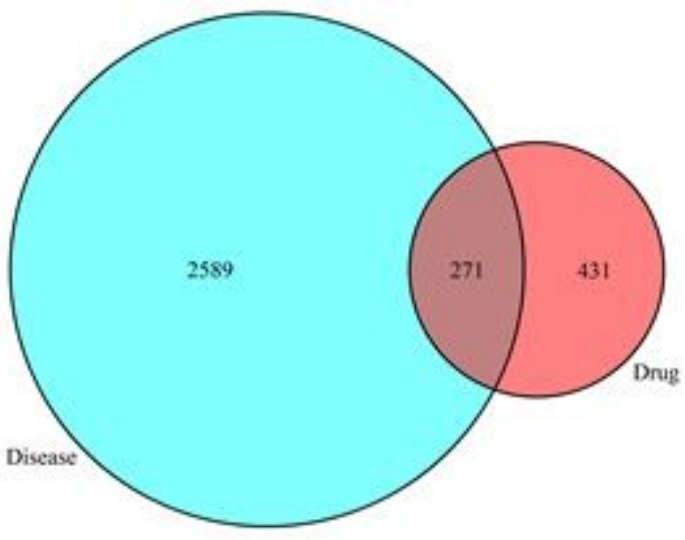

Figure 2

The venn diagram of Triptolide and CTD-ILD targets 

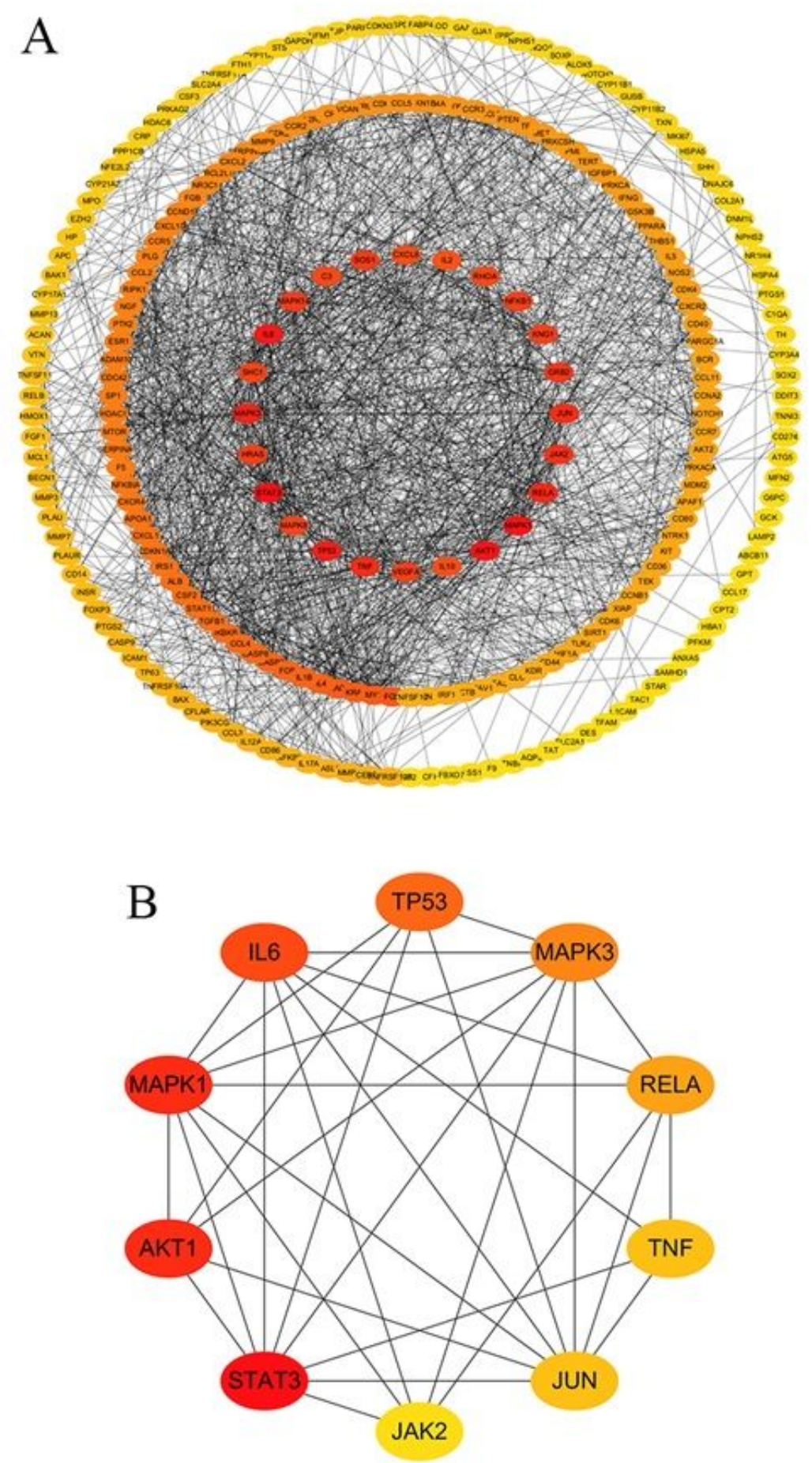

\section{Figure 3}

Against CTD-ILD targets network of Triptolide, the target genes are sorted according to degree. (A). The degree of the outermost circle is 0-20 (purple), the middle circle 21-40 (red), smallest circle 41-100 (yellow). (B).Cytohubba, the plug-in of Cytoscape,was used to analysis the top 10 hub gene network of target proteins by MCC algorithm, in which red and yellow color represents the importance in the network. 
A

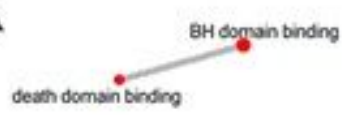

9ycosamiogobican binding

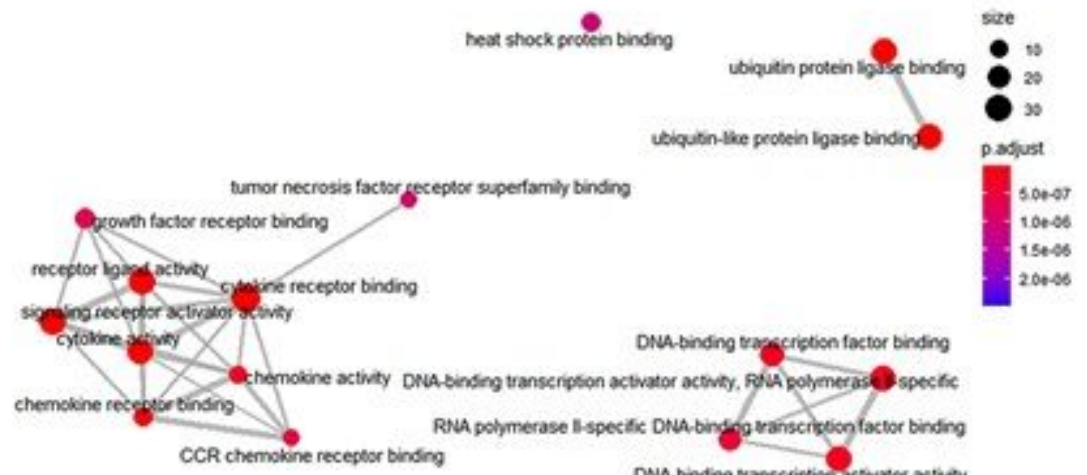

B

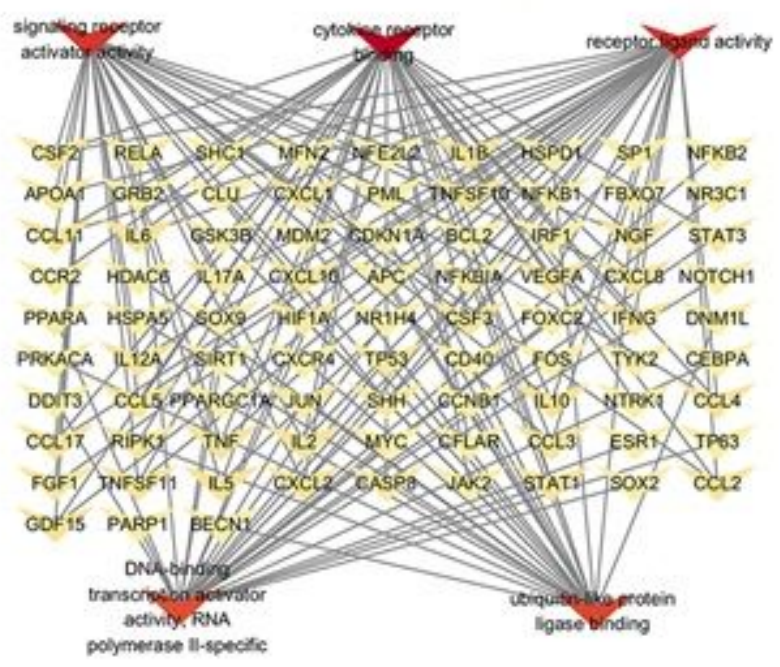

Figure 4

Topological network schematic of proteins targeted by Triptolide and associated with CTD-ILD. (A) Interaction networks between enriched molecular functions analyzed by enrichMap in the clusterProfiler package. The color scales indicated different thresholds of adjusted p-values, and the sizes of the dots represent the gene count of each term. (B) Sub-network showing important genes in the top $5 \mathrm{GO}$ terms. The subnetwork depicts the relationships among $5 \mathrm{GO}$ terms and CTD-ILD associated genes. Abbreviations: GO, gene ontology. 

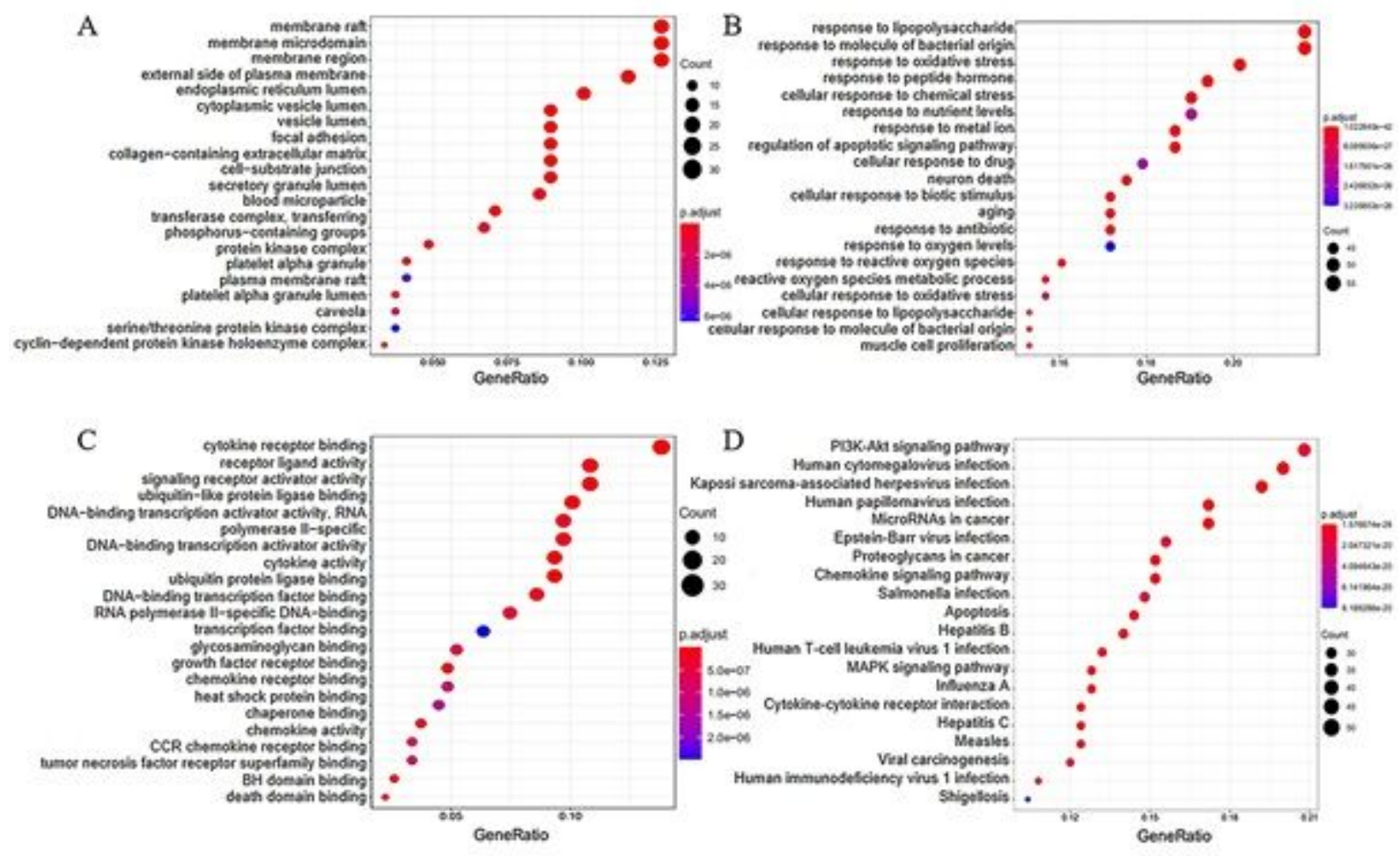

\section{Figure 5}

GO and KEGG analysis of genes encoding proteins targeted by Triptolide. $G O$ enrichment analysis identified genes involved in (A) GO-CC analysis, (B) GO-BP analysis, (C) GO-MF Analysis, (D) KEGG analysis.(B) KEGG pathway analysis from bioinformatics data for the molecular signal pathway of Triptolide against CTD-ILD. 

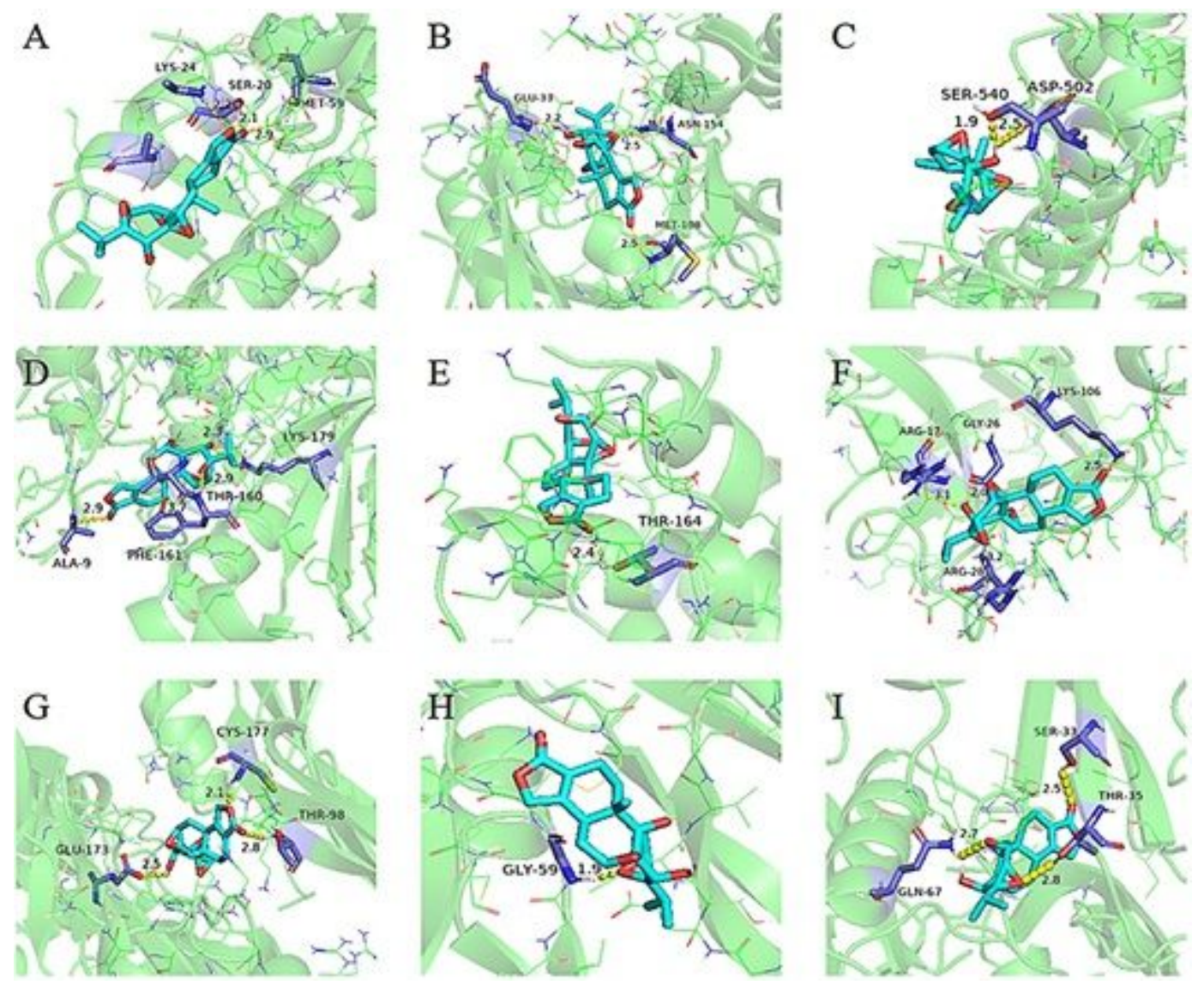

Figure 6

Molecular models of Triptolide binding to its predicted protein targets. Proteins (A)TP53(3DAB), (B) MAPK1(3W55), (C)STAT3(6NUQ), (D)AKT1(6NPZ), (E)IL6(2IL6), (F)FGF(2K8R), (G)PDGF(3MJK), (H) VEGF(1VPF), (I)TGF(3KFD) are shown interacting with a Triptolide molecule, represented by a blue stick model. Lines represent residues in the binding sites. The yellow dashed lines represent hydrogen bonds, the red dashed lines demarcate $\pi-\pi$ interactions, and the interaction distances are indicated next to the bonds. 


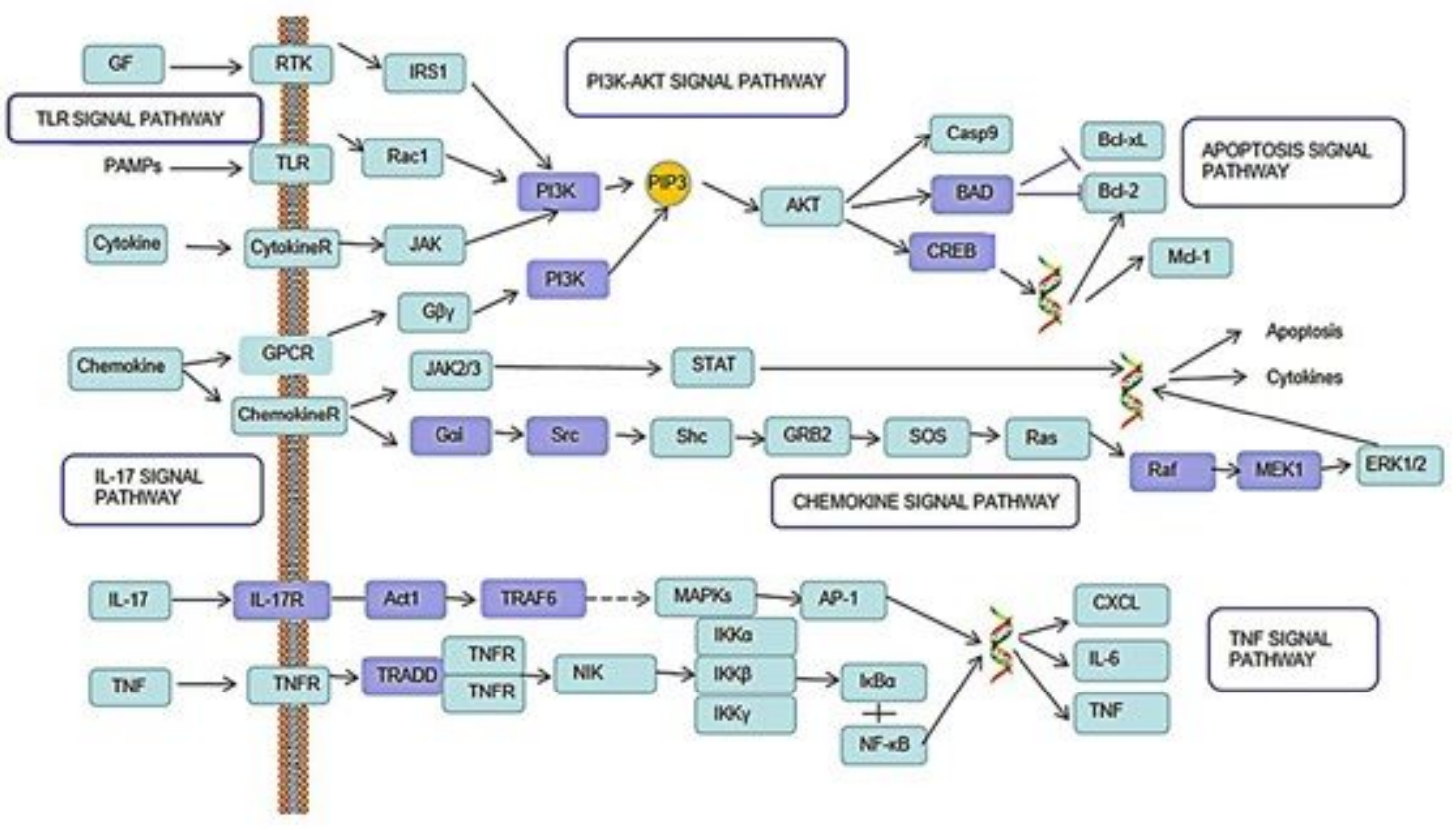

Figure 7

Distribution of the target proteins of Triptolide on the predicted pathway. The purple nodes are potential target proteins of Triptolide, while the blue nodes are relevant targets in the pathway. 